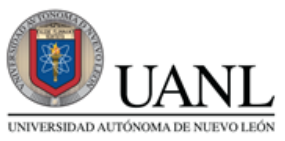

FACPYA
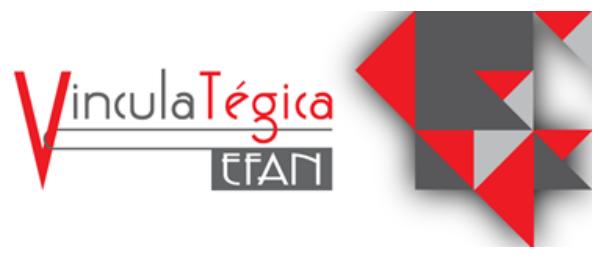

\title{
Factores psicosociales que impactan en el estrés organizacional: primera aproximación teórica
}

\author{
Juan Carlos Flores Aguilar ${ }^{1}$ \\ ${ }^{1}$ Universidad Autónoma de Nuevo León, juan.floresag@uanl.edu.mx, Pedro de Alba S/N, Niños Héroes, \\ Ciudad Universitaria, San Nicolás de los Garza, N.L., Teléfono: 8183-29-4080
}

\author{
Información del artículo revisado por pares \\ Fecha de aceptación: junio-2021 \\ Fecha de publicación en línea: diciembre-2021 \\ DOI: https://doi.org/10.29105/vtga7.2-52
}

\section{RESUMEN}

En los últimos años se han tenido cambios significativos en los entornos económicos, políticos, sociales y organizacionales a nivel global, los cuales han impactado en la vida diaria y en la salud de los individuos en los núcleos familiares, sociales y laborales. El estrés es un problema de salud pública que ocasiona diversos trastornos psicológicos, fisiológicos y emocionales.

En el contexto organizacional el estrés repercute a su vez, en los principales indicadores de negocio en las organizaciones públicas y privadas, por lo cual ha llamado la atención de investigadores, instituciones y gobierno para estudiar su origen, los factores que ocasionan e impactan en el trabajador y el entorno organizacional.

Este estudio se realizó con la finalidad de describir en una primera aproximación teórica, los factores psicosociales que impactan el estrés en las organizaciones. Para ello, se utilizó una metodología documental basada en el uso de fuentes secundarias; mediante la revisión bibliográfica de artículos científicos por autores y organismos especializados.

Se analizó la fundamentación epistemológica del estrés organizacional, las causas y consecuencias con datos, hechos, modelos y teorías con la finalidad de contribuir en la generación de un modelo que intervenga en el análisis, identificación y prevención del estrés organizacional en el ámbito laboral.

Palabras clave: Autonomía, bienestar, entorno, estrés.

Códigos JEL: I310 Bienestar general; Bienestar

\section{ABSTRACT}

In recent years there have been significant changes in the global economic, political, social and organizational environments, which have had an impact on the daily life and health of individuals in family, social and work environments. Stress is a public health problem that causes various psychological, physiological and emotional disorders.

In the organizational context, stress has an impact on the main business indicators in public and private organizations, which is why it has attracted the attention of researchers, institutions and government to study its origin, the factors that cause and impact on the worker and the organizational environment.

This study was carried out with the purpose of describing in a first theoretical approach, the psychosocial factors that impact stress in organizations, For this purpose, a documentary methodology based on the use of secondary sources was used; by means of a bibliographic review of scientific articles by specialized authors and organizations. 
The epistemological foundation of organizational stress, its causes and consequences were analyzed with data, facts, models and theories in order to contribute to the generation of a model that intervenes in the analysis, identification and prevention of organizational stress in the workplace.

Key words: Autonomy, well-being, industrial organization, stress.

JEL Codes: I310 General wellbeing; Wellbeing

\section{INTRODUCCIÓN}

En las organizaciones existen diversos agentes y factores estresantes, los cuales se definen como "situaciones desencadenantes del estrés y pueden ser cualquier estímulo, externo o interno que, de manera directa o indirecta, propicie la desestabilización en el equilibrio dinámico del organismo" (Crespo et al., 2017), pueden ser adaptados de alguna manera por los trabajadores, sin embargo, cuando las estadísticas indiquen una baja de productividad, rotación de personal y los comentarios de los mismos colaboradores mediante el resultado de la aplicación de encuestas de clima laboral o la presencia notoria de un entorno conflictivo donde se perciba una cultura laboral es propicio para determinar que existe niveles de estrés elevados dentro de una organización.

Las legislaciones laborales en México por medio de la STPS determinaron la implementación de la NOM-035 (STPS, 2018) con el fin de identificar, analizar y prevenir los factores de riesgo psicosociales en el trabajo, así como realizar acciones para promover un entorno organizacional favorable, por lo que las organizaciones deberán contar con el conocimiento y aplicación de la normativa laboral que entró en vigencia a partir del 23 de octubre del 2018.

Para precisar los elementos que impactan en el estrés organizacional, se presenta el estudio con el objetivo de describir una primera aproximación teórica de los factores psicosociales que impactan en el estrés, relacionando las variables que originan el estrés en las organizaciones con elementos como el análisis e identificación de las cargas de trabajo, la autonomía para la toma de decisiones y el entorno organizacional.

\section{MARCO TEORICO}

En la época de los 50's del siglo pasado, se empezó a observar las respuestas fisiológicas que generaba el estrés en el organismo; uno de los mayores precursores de la investigación en el tema fue el médico Hans Seyle, el cual describió la sintomatología del paciente que el estrés representaba (Seyle, 1956). Los estudios de Seyle analizaban afectaciones psicológicas y fisiológicas como en el aparato digestivo como diarrea, espasmos, ardores de estómago, náuseas; en el aparato digestivo tal como taquicardia e hipertensión arterial; en el aparato respiratorio como incremento en el ritmo respiratorio, y algunos otros síntomas como tensión dolorosa, tics nerviosos, caída del cabello, etc. (Gallego, Gil, Sepulvéda, 2018).

El médico Seyle, desarrolló desde el año 1926 los primeros modelos teóricos referentes al estrés, donde explicaba que el estrés cuenta con tres fases que describen su aparición. La primera fase de alarma consiste en la percepción del individuo de una posible situación de estrés, donde el organismo comienza a desarrollar una serie de trastornos de orden psicológico y fisiológico, que lo predisponen para enfrentar la situación estresante. La segunda fase, denominada resistencia, se caracteriza por desarrollar un conjunto de procesos emocionales cognitivos y fisiológicos destinados a "negociar" la situación de estrés de la forma menos nociva para el individuo. En la tercera fase, llamada agotamiento, es la fase donde si los mecanismos de adaptación no resultan eficaces se genera un proceso de agotamiento, en el cual los trastornos psicológicos, fisiológicos y/o psicosociales tienden a ser crónicos o irreversibles (Zuñiga, 2019).

En años recientes, la Organización Internacional del Trabajo (OIT) ha señalado el impacto de los riesgos psicosociales, y el estrés organizacional ha recibido más atención entre los investigadores, los especialistas, gobierno y organizaciones. Factores como una mayor competencia en el mercado 
empresarial, expectativas elevadas de productividad que implican largas jornadas laborales, contribuyen a generar un ambiente laboral más estresante (Organización Internacional del Trabajo, 2016).

En octubre del 2018 se oficializado la NOM-035-STPS-2018 (STPS, 2018), la cual tiene como propósito establecer los elementos para prevenir e identificar los factores de riesgo psicosocial y promover un entorno organizacional favorable. Uno de los objetivos de la NOM 035 es establecer límites que eviten jornadas de trabajo superiores a las previstas en la Ley (DOF, 2019), adoptar medidas de prevención y control para prevenir factores de riesgo psicosociales, medir las relaciones dentro del trabajo, el control y las cargas de trabajo, así como establecer un equilibrio entre trabajo-familia mediante evaluaciones y programas de intervención.

De acuerdo a lo publicado en el Comité Mixto OIT-OMS (1984) se presenta en la siguiente figura los factores humanos y del medio ambiente de trabajo que están en interacción continua. Las tareas, el medio ambiente de trabajo, y los factores propios de la organización son representativos de las condiciones laborales. Por su parte, las reacciones de los trabajadores acorde a sus capacidades, necesidades, expectativas, cultura y de vida podría generar una situación psicosocial en el trabajo que tendría repercusiones sobre la salud (ver figura 1).

\section{Figura 1. Factores psicosociales en el trabajo}

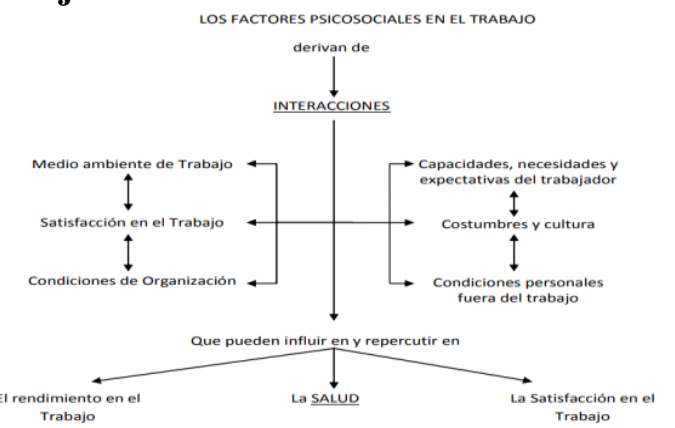

Fuente: Los factores psicosociales en el trabajo (OIT-OMS, 1984)

En el contexto actual en nuestro país, en el año 2012 el Centro estatal de vigilancia epidemiológica y control de enfermedades señaló que México es el segundo país a nivel mundial con el índice más alto en incidencia en estrés organizacional, superado sólo por China (CEVECE, 2014).

Parar el año 2014, el Instituto Mexicano del Seguro Social (IMSS) confirmó con una encuesta realizada por la empresa Regus a México situado en la parte más alta del ranking mundial, superando por vez primera a China; el resultado es que $75 \%$ de las personas que padecen estrés en México señala el entorno laboral como origen, cifra seguida por el $73 \%$ en el caso de China, y cifra lejana por Estados Unidos, con un 59\% (Cruz et al., 2016).

\section{Causas y consecuencias}

Los factores de estrés psicosociales presentes en el trabajo o que son consecuencia de las condiciones de empleo pueden presentarse de forma eventual, de manera continua o persistir durante mucho tiempo. Independientemente del tiempo o de la forma en que se manifiesten los agentes estresores, una persona puede utilizar sus propios recursos para hacer frente a la situación, sin embargo, las exigencias de trabajo pueden rebasar la capacidad del individuo, y la manera de afrontamiento puede no ser eficaz o generar a largo plazo otra serie de problemas (Kalim et al., 1988).

Las consecuencias del estrés organizacional pueden considerarse como perturbaciones de las funciones psicológicas y fisiológicas de un colaborador, así como tener repercusiones que afecten la productividad y rentabilidad de una organización.

\section{Causas:}

1. Cargas excesivas de trabajo.

2. Falta de autonomía en la toma de decisiones.

3. Entorno organizacional.

Consecuencias: Factores que ocasionan el estrés organizacional.

El estrés consiste en un proceso amplio de respuestas por parte del individuo, entre las cuales se encuentran las reacciones emocionales como preocupación, ansiedad, 
angustia, sensación de pérdida de control, la tensión muscular, la sudoración, el malestar digestivo, etc. (Tabares \& Health, 2010). Como consecuencia del estrés, muchos organismos manifiestan reacciones no solo fisiológicas, también psicológicas como ansiedad y depresión.

El estrés organizacional representa una serie de consecuencias negativas para las empresas e instituciones, no solo en las consecuencias directas, como la falta de motivación y satisfacción, baja productividad, así como la ausencia de compromiso por parte del empleado.

Durán (2010) asocia el estrés organizacional con un incremento de ausentismo, accidentes, incapacidades y enfermedades laborales. Genera más conflictos interpersonales entre el trabajo y la familia, mayor índice de errores que influye en la toma de decisiones dentro del entorno laboral, propicia un bajo rendimiento ocupacional, un distanciamiento afectivo con el círculo cercano al trabajador como lo son clientes y proveedores, aumento en los gastos en materia de salud y rotación de personal, entre otros aspectos.

Luceño, et al., (2004) mencionan los costos directos que representa las consecuencias directas del estrés organizacional, como los accidentes de trabajo, las bajas de personal, las pensiones por invalidez; y los costos indirectos, como los relacionados con la disminución del desempeño, el ausentismo y la rotación de personal.

Algunas investigaciones muestran evidencias que indican a los factores psicosociales del entorno laboral como un factor de riesgo de desarrollar enfermedades cardiovasculares (Luceño et al., 2005).

\section{Planteamiento del problema: antecedentes teóricos.}

El estrés organizacional ha sido un tema de interés en la investigación en las ciencias sociales y ciencias de la salud, debido a la importancia que tiene por las consecuencias en la salud que ocasiona, los altos costos que representa y la alta incidencia de trabajadores que lo padecen.

De acuerdo estudio denominado "Manejo adecuado del estrés y programa personal de autocuidado para las y los trabajadores del Instituto Nacional de Desarrollo Social (Indesol)" en el 2010, datos estadísticos relacionados con el estrés son: uno de cada cuatro personas sufre de falta de sueño producto del estrés; entre el $75 \%$ y el $90 \%$ de las visitas a consultas médicas por parte de personas adultas están relacionadas con trastornos de estrés, y el $89 \%$ de las personas adultas aseguran tener "altos niveles de estrés"(SEDESOL. Gobierno Federal, 2012).

Según un estudio elaborado por la OIT, el estrés organizacional podría suponer pérdidas de entre el $0.5 \%$ y el $3.5 \%$ del PIB de los países. Si bien existe escasos datos estadísticos sobre las pérdidas económicas que el estrés organizacional produce en México, supondrían unas pérdidas aproximadas de entre 5.000 y 40.000 millones de dólares al año (Cruz et al., 2016).

En este estudio, se describen antecedentes teóricos de las variables independientes: cargas de trabajo, autonomía para la toma de decisiones, y entorno organizacional, siendo la variable dependiente el estrés organizacional. Se mencionan autores, organizaciones, teorías y modelos de cada una de las variables independientes a lo largo del tiempo, que han impactado en el estrés organizacional.

\section{Cargas de trabajo}

Algunos teóricos como O’Donell \& Eggemeier (1986) señalan que el término de carga de trabajo implica la complicación para la realización de las tareas principales, y si las demandas del procesamiento de una o varias de ellas exceden la capacidad existente, se tendrá como consecuencia, aparte del estrés organizacional, un decremento en el desempeño individual y una afectación organizacional. En el año 1990, Karasek y Theorell, consideraron que la sola carga de trabajo mental es un factor desencadenante de estrés, debido a que constituye uno de los factores más significativos que pueden determinar la presencia de estrés en el 
trabajador (González E. \& Gutiérrez R., 2006).

Existe otro termino, relacionado a las cargas excesivas de trabajo definido como Burnout, o de desgaste profesional; fue acuñado en 1974 por Freudenberger en referencia al resultado de la relación establecida entre el individuo y su trabajo, la cual conduce al cansancio emocional y físico, debido a las condiciones laborales. La definición más aceptada del Síndrome de Burnout es la definida por Maslasch \& Jackson (1981), ellos consideran que tal síndrome es una inadecuada forma de afrontar un estrés emocional crónico, siendo las características principales, la despersonalización, el agotamiento emocional y la disminución del desempeño personal (Méndez, 2004).

La Organización Mundial de la Salud en el año 2001, reconoció el Síndrome de Burnout como riesgo de salud ocupacional para profesionales de la salud, educación y servicios asistenciales, que conduce al malestar físico y psicológico comprometiendo los resultados en el trabajo, repercutiendo negativamente en las organizaciones debido al ausentismo, aumento de los conflictos interpersonales e incremento en la rotación de personal (Barrios, et al., 2012).

Siguiendo con el concepto de cargas de trabajo, en varios países de economías medias y bajas tienen un efecto acelerado de modernización del sector empresarial.

Particularmente en México, este proceso implica que los trabajadores enfrenten condiciones laborales que modifican las demandas requeridas del desempeño, las cuales pasan de un elemento físico a actividades que implican a los empleados una mayor carga de trabajo mental. Esta se caracteriza por tareas repetitivas con demandas altas de atención, originando una alta concentración por parte del trabajador y poco control sobre las tareas que realiza, provocando también efectos negativos en la salud mental de los trabajadores (González E. \& Gutiérrez R., 2006).

La STPS (2018) define como carga de trabajo a "las exigencias que el trabajo impone al trabajador y que exceden su capacidad, pueden ser de distinta naturaleza, como cuantitativas, emocionales, cognitivas, de responsabilidad, así como cargas contradictorias o inconsistentes"

\section{Autonomía para la toma de decisiones}

La OIT (2016) define el término de autonomía para la toma de decisiones como la participación en la toma de decisiones, el control sobre el trabajo (particularmente en forma de participación, como cuestión organizativa). La falta de autonomía es una característica estresante correspondiente a los factores de riesgo psicosocial; por el lado opuesto, con pequeñas dosis de autonomía en la realización de las tareas es suficiente para tener beneficios en la salud mental y la productividad de los trabajadores.

Una de las teorías referente al estrés organizacional mencionada por Moreno et al. (2005), es el modelo Demanda-Control donde se encuentran dos características relacionadas en común para generar estrés laboral, la primera es las demandas de las tareas y el control que se tiene sobre las mismas como segunda característica. Las altas demandas laborales son elementos que ocasionan exceso de trabajo, falta de tiempo y en priorización para cumplir con las actividades requeridas.

Por otra parte, los resultados en distintas aplicaciones del cuestionario basado en el modelo de interacción entre Individuo y Organización de Leiter y Maslach, señala al control en las tareas o en otras palabras, la autonomía de actividades, como el segundo factor más mencionado en la literatura científica del estrés laboral (solo debajo de las cargas de trabajo), la cual se define como la oportunidad de realizar elecciones y tomar decisiones, resolver problemas, y contribuir al cumplimiento de las responsabilidades (Rodríguez \& Rivas, 2011).

Las demandas de la tarea y las demandas en el papel son componentes que pueden ocasionar estrés organizacional. Las demandas de la tarea corresponden a elementos de diseño del puesto de trabajo, como la autonomía, la variedad de actividades, 
y el grado de automatización; mientras mayor sea la interdependencia de un colaborador, mayor será el estrés; por el contrario, la autonomía en la toma de decisiones puede disminuirlo. Las demandas en papel son las presiones que puede tener un empleado en función de su rol en una organización; los conflictos de rol y la falta de claridad en las tareas asignadas provocan conflictos en el trabajador respecto a las funciones $y$ responsabilidades a desempeñar (Atalaya, 2001).

Las demandas de una tarea son factores que se relacionan a las actividades de una persona respecto al diseño del trabajo dentro de una estructura de funciones de puesto, tales como el grado de autonomía y la variedad de actividades. Atalaya, (2001) comenta que cuanto mayor sea la interdependencia de actividades de una persona, mayor será el estrés potencial, por lo que la autonomía tiende a disminuir el estrés. La falta de participación en la toma de decisiones es por sí misma, una de las causas que origina el estrés laboral.

\section{Entorno organizacional}

Algunas fuentes potenciales del estrés organizacional corresponden a la estructura y el liderazgo organizacional. En el caso de la estructura organizacional si esta cuenta con alta burocracia en sus procesos y exceso de reglamentos es una fuente probable de estrés. En el aspecto del liderazgo organizacional, algunos líderes, crean una cultura de temor, tensión y ansiedad ocasionadas por metas inalcanzables, exceso control e incluso despidos a empleados por desempeño (Atalaya, 2001).

Las relaciones con jefes, subordinados y/o compañeros pueden ser una causa de estrés organizacional. La relación con compañeros de trabajo es una variable habitualmente muy valorada en el entorno organizacional y un elemento fundamental en la salud emocional personal; el apoyo social percibido supone un amortiguador de las experiencias estresantes, por el contrario, la ausencia de contacto con otros trabajadores o la ausencia de colaboración entre los mismos puede producir niveles elevados de tensión y estrés entre los miembros de un equipo de trabajo. Un estilo de liderazgo determinado tanto de jefes y/o colaboradores, también es un ingrediente en el grado de satisfacción o de tensión en el tema de supervisión del personal (Casas et al., 2002).

Concerniente a las instituciones, la experiencia de la OIT muestra que el éxito de una organización se basa en sus trabajadores y en su cultura organizativa. Los trabajadores que se encuentran en un entorno seguro y propicio se sienten mejor y por lo general están más sanos, lo cual se traduce a su vez en un menor ausentismo, mayor motivación, mejora de la productividad y una imagen positiva de la organización ante la sociedad (Organización Internacional del Trabajo, 2016).

Además, la OIT menciona que un trabajo saludable es aquel en que la presión sobre el empleado se corresponde con sus recursos y capacidades, el grado de control que ejerce sobre su actividad y el apoyo que recibe de otros compañeros. Dado que la salud se considera un estado de completo bienestar físico, mental y social, y no solamente la ausencia de afecciones o enfermedades (Kalim et al., 1988), un entorno saludable no es únicamente aquel característico de ausencias de elementos perjudiciales, sino abundancia de factores que promuevan la salud (Leka \& Griffiths, 2004).

En el marco jurídico en México, el propósito establecido por el Reglamento Federal de Seguridad y Salud en el Trabajo, con la incorporación de los factores de riesgo psicosocial en el año 2014, consiste en establecer elementos para identificar, analizar y prevenir los factores de riesgo psicosocial, así como promover un entorno organizacional favorable en los centros de trabajo (Moreno, 2018).

\section{METODO}

El diseño de la presente investigación es de tipo transeccional con el propósito de describir variables y analizar la incidencia e interrelación de las variables independientes con la variable dependiente (Hernández, R., Fernández, C., Baptista, 2014); utilizando 
técnicas de investigación documental y bibliográfica.

El estudio realizado considera elementos descriptivos con la finalidad de identificar hechos, situaciones, rasgos, características del objeto de estudio, correlacional en el método para analizar las causas y efectos de la relación entre variables (Bernal, 2006), de igual manera, dar a conocer los factores que impactan el estrés organizacional; y explicativo con el objetivo de explicar la ocurrencia del fenómeno, en qué condiciones se manifiesta y por qué se relacionan dos o más variables en la investigación (Hernández, et al. 2014).

La perspectiva teórica de la investigación se encuentra realizada en un contexto organizacional, social, académico y científico, tomando en consideración, los antecedentes, los hechos vigentes y las expectativas en materia laboral, social y de salud del estrés organizacional a nivel nacional e internacional.

\section{CONCLUSIONES}


Las aportaciones teóricas describieron variables correspondientes a las causas del estrés organizacional, la afectación hacia la salud del trabajador en su entorno social y laboral, así como de las organizaciones, al influir en indicadores de productividad y desempeño. La aportación práctica consistió en generar concientización de los principales factores que impactan el estrés laboral desde una primera aproximación, con la finalidad realizar una investigación posterior de tipo cuantitativo aplicando instrumentos de medición que permitan evaluar la relación de las variables de forma metodológica.

Los límites espaciales se ubicaron en México acorde a la legislación en materia laboral vigente, posteriormente se realizará una investigación de campo en el área metropolitana de Nuevo León. Los límites temporales son de tipo transeccional; siendo los límites teóricos los modelos, datos y acontecimientos encontrados hasta el momento del presente estudio.

El estudio a su vez, describió los factores psicosociales que impactan el estrés laboral en su primera aproximación teórica, en el cual se detallaron hechos, antecedentes del estrés y sus consecuencias, y los modelos teóricos que se han desarrollado a lo largo del tiempo en el estudio del estrés organizacional, las causas y consecuencias, así como la relación de factores psicosociales que impactan el estrés en las organizaciones.

Por una parte, las cargas de trabajo es uno de los factores más estudiados y analizados en diversas investigaciones, organismos especializados a nivel internacional y en años recientes incorporado en las legislaciones laborales como factor de riesgo psicosocial.

Las cargas de trabajo se pueden presentar de manera física y mental, tienen relación con otros factores descritos en este estudio como la autonomía en la toma de decisiones, al no tener control sobre las actividades por falta de las especificaciones escritas o por el tipo de liderazgo de los supervisores; y el entorno organizacional puede contener un clima negativo, seguido de la falta de procesos o manuales de puesto que ayuden al colaborador a tener definidos los procedimientos funcionales en su actividad diaria.

Por otra parte, la autonomía en la toma de decisiones es un factor relacionado con el resto de los factores psicosociales, se considera un elemento positivo cuando el empleado tiene la facultad de tomar decisiones previamente informado de las especificaciones, límites de las responsabilidades y previamente capacitado para ejercer sus funciones en el puesto de trabajo.

Finalmente, el entorno organizacional es otro factor que se analizó en esta investigación. El entorno de trabajo impacta en diversos sentidos para el generar estrés laboral, cuando la organización tiene lideres con una influencia negativa hacia sus colaboradores, o bien cuando no existen adecuados procesos para resolver situaciones cotidianas que ocurren durante la actividad diaria del empleado. Sin embargo, contar con un entorno laboral positivo, influye en satisfacción laboral, motivación y el bienestar en la calidad de vida los trabajadores.

En la actualidad, favorablemente cada vez son más las organizaciones que cuentan con planes estratégicos de responsabilidad social y están comprometidas con el cumplimiento de las normas que promuevan el entorno favorable para sus colaboradores.

Las recomendaciones finales para investigaciones futuras son, sensibilizar a los especialistas, investigadores, y organizaciones respecto a las consecuencias del impacto del estrés laboral como objeto de estudio; del mismo modo, elaborar instrumentos de medición de las variables mencionadas como las cargas de trabajo, la autonomía para la toma de decisiones y la evaluación del entorno organizacional para analizar los resultados con el estrés organizacional, que permitan realizar planes de acción para mejorar el entorno organizacional, el bienestar y la calidad de vida de los trabajadores.

\section{REFERENCIAS}


Atalaya, M. (2001). El Estrés Laboral y su influencia en el trabajo. In Industrial Data (Vol. 4, Issue 2, pp. 25-36). http://revistasinvestigacion.unmsm.edu.pe/index.php/idata/article/view/6754

Barrios, S., Arechabala, M., \& Valenzuela V. (2012). Relación entre carga laboral y burnout en enfermeras de unidades de diálisis. Enfermería Nefrológica, 15(1), 46-55. https://doi.org/10.4321/s2254-28842012000100007

Bernal, C. (2006). Metodología de la Investigación.

Casas, J., Repullo, J., \& Lorenzo, S. (2002). Estrés laboral en el medio sanitario y estrategias adaptativas de afrontamiento. Revista de Calidad Asistencial, 17(4), 237-246. https://doi.org/10.1016/S1134-282X(02)77511-8

CEVECE. (2014). Estrés laboral. 1-7.

Comité Mixto OIT-OMS. (1984). Factores psicosociales en el Trabajo: naturaleza, incidencia y prevención. Oficina Internacional Del Trabajo, 1, 1-85. http://biblioteca.uces.edu.ar/MEDIA/EDOCS/FACTORES_Texto.pdf

Crespo, B., León, M., García-Unanue, J., Gallardo, L., \& Maicas, L. (2017). Estudio de Caso de Éxito: Implementación de Tecnología para La Gestión del Estrés en El Entorno Laboral. PODIUM Sport, Leisure and Tourism Review, 6(3), 57-71. https://doi.org/10.5585/podium.v6i3.223

Cruz, M., López, E., Cruz, R., \& Llanillo, M. (2016). El estrés laboral en México. Tópicos Selectos de Micro y Pequeñas Empresas, 1, 368-376. http://www.ecorfan.org/actas/A_1/32.pdf

DOF. (2019). Ley Federal del Trabajo. Ley Federal Del Trabajo, Última ref, 1-235.

Durán, M. (2010). Bienestar Psicológico: El Estrés Y La Calidad De Vida En El Contexto Laboral. Revista Nacional de Administración, 1(1), 71-84. https://doi.org/10.22458/rna.v1i1.285

Gallego, Y., Gil, S., Sepulvéda, M. (2018). Revisión teórica de eustrés y distrés definidos como reacción hacia los factores de riesgo psicosocial y su relación con las estrategias de afrontamiento. Tesis, 151(2), 10-17.

González E. \& Gutiérrez R. (2006). La carga de trabajo mental como factor de riesgo de estrés en trabajadores de la industria.

Hernández, R., Fernández, C., Baptista, P. (2014). Metodología de la investigación (M. G. H. Education (ed.); 6a.).

Kalim, R., El-Batawi, M., \& Cooper, C. (1988). Los factores psicosociales en el trabajo y su relación con la salud. Organización Mundial de La Salud, 106. http://apps.who.int/iris/bitstream/10665/37881/1/9243561022_spa.pdf

Leka, S., Griffiths, A. (2004). La organización del trabajo y el estrés.

Luceño, L., Martín, J., Díaz, E., \& Rubio, S. (2004). Factores psicolsociales en el entorno laboral, estrés y enfermedad. EduPsykhé: Revista de Psicología y Psicopedagogía, 3(1), 95-108.

Luceño, L., Martín, J., Jaén, M., \& Díaz, E. (2005). Evaluación de factores psicosociales en el entorno laboral. EduPsykhé, 4(1), 19-42.

Maslach, C., \& Jackson, S. (1981). The measurement of experienced burnout. Journal of Organizational Behavior, 2(2), 99-113. https://doi.org/10.1002/job.4030020205

Méndez, J. (2004). Estrés laboral o síndrome de "burnout.” Acta Pediátrica de México, 25(5), 299302.

Moreno, J. (2018). Marco legal de los factores de riesgo psicosocial. Primer Jornada Del IMSS Sobre Factores de Riesgo Psicosociales. Estrés y Salud Mental En El Trabajo, 1-35. http://www.imss.gob.mx/sites/all/statics/salud/estreslaboral/1erjornada/02-Marco-Legal.pdf

O’Donell, R. \& Eggemeier, F. (1986). Workload assessment methodology. Vol. II Cognitive Processes and Performance. Nueva York: Wiley. http://apps.usd.edu/coglab/schieber/docs/odonnell.pdf

Organización Internacional del Trabajo. (2016). Estrés en el trabajo: un reto colectivo. In Gestión de las Personas y Tecnología (Vol. 9, Issue 25). https://www.ilo.org/public/libdoc/ilo/2016/490658.pdf

Rodríguez, R., \& Rivas, S. (2011). Los procesos de estrés laboral y desgaste profesional (burnout): 
diferenciación, actualización y líneas de intervención. Medicina y Seguridad Del Trabajo, 57, 72-88. https://doi.org/10.4321/s0465-546x2011000500006

SEDESOL. Gobierno Federal. (2012). Pausas para la Salud: Manejo adecuado del estrés y programa personal de. Instituto Nacional de Desarrollo Social (Indesol), 1-36.

https://www.gob.mx/cms/uploads/attachment/file/160956/13._cuaderno_pausas_para_la_salud .pdf

Seyle, H. (1956). The Stress of Life. Hans Selye, M.D. New York, McGraw-Hill. 1957. https://journals.lww.com/jbjsjournal/Citation/1957/39020/The_Stress_of_Life_Hans_Selye, M_D_New_York,.34.aspx

STPS. (2018). Norma Oficial Mexicana NOM-035-STPS-2018, Factores de riesgo psicosocial en el trabajo-Identificación, análisis y prevención. 1-54. http://sidof.segob.gob.mx/notas/5541828

Tabares, A., \& Health, A. (2010). Imago mundi: la contribución cultural de la medicina al estrés urbano. Crítica, 968(August), 4.

Zuñiga, E. (2019). El Estrés Laboral y su Influencia en el Desempeño de los Trabajadores. Gestión En El Tercer Milenio, 22(44), 115-120. https://doi.org/10.15381/gtm.v22i44.17317 